increase in $\mathrm{Li}^{+}$mobility at ambient temperatures compared with conventional 'salt-in-polymer' electrolytes, a way has been opened to a new generation of lithium batteries with high-power density applications in prospect.

Equally important now that such a rise in ionic mobility has been achieved without the use of volatile 'plasticizers' such as propylene carbonate, a class of materials emerges which may be ther- mally stable up to $150^{\circ}$ or even $200{ }^{\circ} \mathrm{C}$. These electrolytes could therefore bridge the present gap in battery technology between essentially ambient-temperature devices which operate up to $100{ }^{\circ} \mathrm{C}$ and the high-temperature molten salt systems.

Malcolm Ingram is in the Department of Chemistry, University of Aberdeen, Meston Walk, Old Aberdeen AB9 2UE, UK.

\title{
Turning on quantum taps
}

\section{Stephen M. Barnett}

Measuring a quantum-mechanical observable should collapse the wavefunction so that all future measurements give the same answer, but in practice the act of observation generally demolishes the original quantum state of the system, leaving no possibility of future measurements. J. Ph. Poizat and P. Grangier (Phys. Rev. Lett. 70, 271-274; 1993) have now circumvented this sticky problem by developing a method of delicately tapping off information on the state of a laser beam whilst leaving the signal unchanged.

Quantum mechanics is fundamentally a probabilistic theory. It is usually the case that we can predict only the probability that a measurement will yield a particular result. If we prepare a large number of copies of our system in the same state then we will find that the results of individual measurements will differ but that the distribution of results will be characteristic of the state prepared. A simple example is the measurement of one component of spin possessed by a spin-half particle. Individual measurements will always give the results $+\hbar / 2$ or $-\hbar / 2$, and the average over many observations will produce the average value of the spin component.

The ideal model of a measurement in quantum theory has two properties. First, it records one of the possible values of the measured observable; second, it changes the state of the system, so that a sequence of ideal measurements carried out on the same system will always produce the same result. For our spin-half particle this means that if one measurement of the $z$-component of the spin leads to the result $+\hbar / 2$ then so will all subsequent measurements.

This theoretical ideal is rarely achieved in experiments, as the act of observation is usually too violent for the quantum state to survive intact. This is especially true in quantum optics. We can count the number of photons in a beam with great accuracy, but the photodiodes used to count the photons also absorb and thus destroy them. We can certainly do the measurement but fall far short of the theoretical ideal of leaving the state with a precisely determined number of photons, ready for repeated observation.

To approach the ideal quantum measurement for light we require a less violent detection process. Suitable processes are referred to as quantum nondemolition (QND) measurements. With a QND detector for light we could measure any information encoded on the amplitude of a laser beam without degrading the quality of the information left on the beam. Such a detector could justly be dubbed a quantum optical tap, in that it allows us to tap into an optical signal and measure the amplitude modulation without changing the signal.

Poizat and Grangier's quantum optical tap works by the nonlinear optical effect of cross-phase modulation. In a suitable nonlinear medium (in this case a sodium cell) the refractive index will be modified by light propagating through it. The index change will be proportional to the intensity of the light, so it leads to an intensity-dependent phase modulation. It is this phase modulation that is responsible for the production of solitons (self-sustaining wave packets) in optical fibres. When two beams are present, the intensity-dependent modification of the refractive index induced by one beam can produce a modulation of the phase in the second beam. This crossmodulation means that the phase change of one beam is proportional to the intensity of the other, so by monitoring the phase of a 'meter' beam which has interacted with the signal beam we can infer the intensity of the signal.

As the signal is not absorbed, it continues to propagate and could in principle be measured again in the same way. Poizat and Grangier in fact measure the signal by conventional means after the QND measurement. Their comparison of these two measurements shows that the signal does not become appreciably depleted, and that the signal and meter beams are correlated so that the measurement carried out on the meter tells us something about the signal amplitude. This new experimental arrangement has achieved the sensitivity required to push the nondemolition measurement into the quantum regime where the signal degradation is lower and the correlation between the meter and signal beams is stronger than could be achieved in any classical arrangement. A conventional measurement would either have to degrade the signal more to achieve the same degree of accuracy in the measurement, or sacrifice accuracy in order to leave the signal intact.

There is, of course, a price to be paid. Measurement of the amplitude scrambles the phase of the signal beam. The nonlinear interaction between the meter and signal beams, causing a correlation between the signal intensity and the meter phase, also correlates the signal phase and the meter intensity. In monitoring the meter phase we discard information about the meter intensity and hence introduce additional uncertainty into the signal phase. At the quantum level this is simply a manifestation of complementarity, in that to measure one observable, the amplitude, we induce an uncertainty in the complementary observable, the phase.

In an interesting variation on the QND theme, Grangier and colleagues also used a nonlinear optical crystal to produce two amplified copies of the information contained in one quadrature component of an input signal (J. A. Levenson et al. Phys. Rev. Lett. 70, $267-270 ;$ 1993). Thus an amplitudemodulated signal is converted into two beams, each more intense than the original and carrying the same amplitude modulation. Observation of the amplitude of one beam reveals the modulation carried by its partner. This is not strictly a QND measurement because the signal after measurement does not have the same amplitude as it had previously. But the fact that it is more intense might make this technique useful in overcoming losses.

Optical QND measurements allow us to approach the ideal quantum measurement, although there is still some degradation of the signal - the quantum optical tap drips. Given time, advances in the development of nonlinear optical materials should provide the necessary washers.

Stephen M. Barnett is in the Department of Physics and Applied Physics, University of Strathclyde John Anderson Building, 107 Rottenrow, Glasgow G4 ONG, UK. 